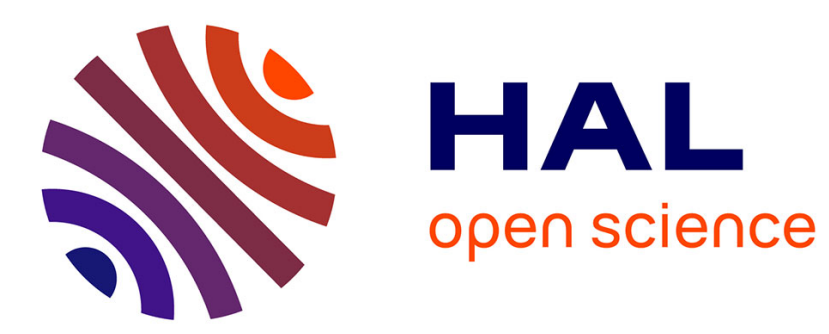

\title{
Variabilité spatio-temporelle des précipitations aux échelles de l'hydrologie urbaine
}

\author{
I. Emmanuel, E. Leblois, H. Andrieu, B. Flahaut
}

\section{To cite this version:}

I. Emmanuel, E. Leblois, H. Andrieu, B. Flahaut. Variabilité spatio-temporelle des précipitations aux échelles de l'hydrologie urbaine. La Houille Blanche - Revue internationale de l'eau, 2011, 4, p. 31 p. 36 . 10.1051/lhb/2011038 . hal-00779396

\section{HAL Id: hal-00779396 https://hal.science/hal-00779396}

Submitted on 22 Jan 2013

HAL is a multi-disciplinary open access archive for the deposit and dissemination of scientific research documents, whether they are published or not. The documents may come from teaching and research institutions in France or abroad, or from public or private research centers.
L'archive ouverte pluridisciplinaire HAL, est destinée au dépôt et à la diffusion de documents scientifiques de niveau recherche, publiés ou non, émanant des établissements d'enseignement et de recherche français ou étrangers, des laboratoires publics ou privés. 


\title{
Variabilité spatio-temporelle des précipitations aux échelles de l'hydrologie urbaine
}

\author{
Isabelle EMMANUEL ${ }^{1}$, Etienne LEBLOIS ${ }^{2}$, Hervé ANDRIEU ${ }^{1}$, Bernard FLAHAUT ${ }^{1}$ \\ Ifsttar-e-mail : isabelle.emmanuel@ifsttar.fr / herve.andrieu@ifsttar.fr / bernard.flahaut@ifsttar.fr \\ 2.CEMAGREF - e-mail : etienne.leblois@cemagref.fr
}

\begin{abstract}
RÉSUMÉ. - Le principal objectif de cet article est de caractériser la variabilité spatiale et temporelle de champs pluvieux à des échelles spatio-temporelles fines cohérentes avec les applications hydrologiques urbaines. Dans cette optique, 24 périodes pluvieuses ont été analysées selon une approche géostatistique. Cette analyse s'est centrée sur le variogramme de la Pluie Non Nulle. Les périodes pluvieuses considérées ont été enregistrées, entre mai et novembre 2009, par le radar météorologique de Treillières. Ce radar bande C est situé à $10 \mathrm{~km}$ au Nord de Nantes (France). Il fournit des images radar de haute résolution spatiale $\left(250 \times 250 \mathrm{~m}^{2}\right)$ et de résolution temporelle instantanée. Cette étude a permis de mettre en évidence quatre types de structures pluvieuses présentant des échelles très différentes de variabilité, dont des doubles structures localisées à l'intérieur d'un même champ pluvieux. Ce travail a montré que les images radar de résolution fine permettaient de capturer des petits amas pluvieux intenses et fortement variables. Ces résultats pourront être utilisés afin d'étudier l'influence de la variabilité de la pluie sur la variabilité des débits au niveau de bassins versants urbains. Couplés à l'analyse des variogrammes d'indicateur, ces résultats pourront être également utilisés à des fins de modélisation de champs pluvieux.
\end{abstract}

Mots-clés : structure des précipitations, géostatistiques, hydrologie urbaine

\section{Temporal and spatial variability of rainfall at urban hydrological scales}

\begin{abstract}
The main objective of this paper is to characterize the spatial and temporal variability of rainfall at spatial and temporal scales that are consistent with urban hydrological applications. In this way, 24 rain periods have been analyzed according to a geostatistical approach. This analysis has focused on the non-zero rainfall variogram. The studied rain periods were recorded by the weather radar of Treillières between May and November 2009. This C-band radar is located $10 \mathrm{~km}$ north of Nantes (France). It provides rainfall radar images of high spatial resolution $\left(250 \times 250 \mathrm{~m}^{2}\right)$ and of instantaneous temporal resolution. Results indicated four different types of rainfall fields, which display very different variability scales, including double structures within the same fields. This study highlights the benefit of radar images featuring high resolution, which in turn allow studying small-scale variability. It would be of interest to analyse how those different rainfall structures affect hydrological modelling of urban watersheds. Associated with the analysis of the indicator variograms, those results could be used to simulate rainfall fields.
\end{abstract}

Key words : rainfall structure, geostatistics, urban hydrology

\section{INTRODUCTION}

L'influence de la variabilité spatiale de la pluie sur la modélisation hydrologique pluie-débit est un sujet de recherche actif, comme en témoigne la littérature abondante publiée ces dernières années ( $c f$. [1]). Les petits bassins versants urbains peuvent être sensibles à la variabilité spatio-temporelle des précipitations. Afin de mieux comprendre le lien existant entre la variabilité de la pluie et la réponse hydrologique des bassins versants urbains, une connaissance détaillée des champs pluvieux apparaît donc utile. Les images de précipitation mesurées par radars météorologiques, caractérisées par une haute résolution spatio-temporelle, semblent un outil tout à fait adapté pour mener à bien des investigations poussées sur ce sujet. Des études récentes ont confirmé l'amélioration de la mesure de pluie par radar météorologique [2] mais ont aussi montré qu'il était nécessaire de corriger le biais significatif affectant les images radar avant leur utilisation [3].

Dans ce contexte, notre travail a pour objectif la caractérisation de la variabilité spatiale et temporelle de la pluie à l'aide de méthodes géostatistiques, approche devenue clas- sique pour caractériser la structure spatio-temporelle des précipitations [4]. Cette étude porte sur l'analyse d'images radar de résolution spatiale particulièrement fine $\left(250 \times 250 \mathrm{~m}^{2}\right)$ et de résolution temporelle instantanée. 13 journées pluvieuses enregistrées entre mai et novembre 2009 par le radar de Treillières (10 km au Nord de Nantes) ont été analysées.

\section{DONNEES UTILISEES}

\section{II.1. Données radar}

Les données radar sont issues du radar météorologique bande $\mathrm{C}$ de Treillières (10 km au Nord de Nantes, France). Son protocole de balayage volumique comprend trois cycles panoramiques successifs. Chaque cycle, d'une durée de 5 min, est constitué d'images à quatre angles de site. Les données polaires sont projetées, toutes les $5 \mathrm{~min}$, sur une grille cartésienne de $128 \times 128 \mathrm{~km}^{2}$, de résolution spatiale de $250 \times 250 \mathrm{~m}^{2}$ (Figure 1). Les données de chaque pixel sont instantanées. 
Les données radar utilisées ont été traitées selon le protocole suivant :

1. Identification dynamique des échos fixes. Il s'agit de déterminer pour chaque pixel si la mesure provient d'un écho fixe ou de la pluie [5].

2. Composition statique, pour chaque cycle, des mesures effectuées aux différents angles de site. La mesure de l'angle de site le plus bas (qui ne soit pas un écho fixe) a été utilisée.

3. Filtrage des pixels isolés. Un pixel est identifié comme isolé dès lors que sa valeur de réflectivité diffère de $20 \mathrm{dBZ}$ de la valeur moyenne de ses huit voisins.

4. Conversion de la réflectivité en taux de pluie en utilisant la relation $Z-R$ de Marshall-Palmer (c'est-à-dire $Z=200 \mathrm{R}^{1.6}$, avec $Z=$ réflectivité radar en $\mathrm{mm}^{6} / \mathrm{m}^{3}$ et $R=$ taux de pluie en $\mathrm{mm} / \mathrm{h}$ ).

5. Invalidation des zones de masque. Trois zones de sousestimation de la pluie ont été invalidées (Figure 1) après visualisation de l'image de cumul de pluie sur les 13 journées pluvieuses.

Pour travailler à une résolution effective de $250 \times 250 \mathrm{~m}^{2}$, la zone d'étude a dû être limitée à un cercle de $20 \mathrm{~km}$ de rayon centré sur le radar de Treillières (Figure 1).

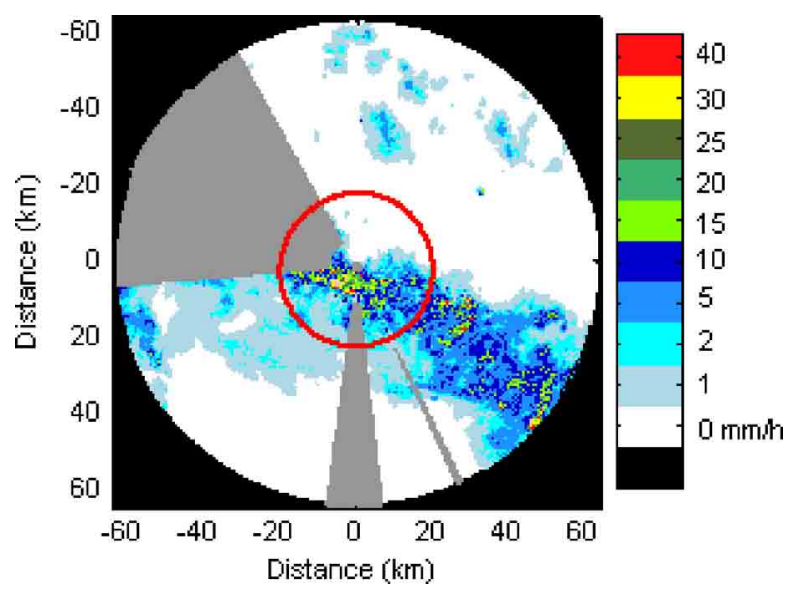

Figure 1: Exemple d'image d'intensité radar en $\mathrm{mm} / \mathrm{h}$ mesurée par le radar de Treillières. Les 3 zones de masque sont indiquées en gris. Le cercle central délimite la zone d'étude considérée (cercle de rayon de $20 \mathrm{~km}$ ).

\section{II.2. Périodes pluvieuses sélectionnées}

13 journées pluvieuses enregistrées entre mai et novembre 2009 ont été considérées. Le cumul de pluie sur la zone d'étude et l'écart-type calculé entre les différents pixels de l'image radar, par journée pluvieuse, montrent que les journées considérées semblent de types très différents. Les cumuls de pluie varient entre 2.2 et $27.5 \mathrm{~mm}$, avec des écarttypes compris entre 1 et $13.6 \mathrm{~mm}$.

\section{II.2.1. Séparation des journées en périodes pluvieuses homogènes et en groupes}

D'après les bulletins climatologiques mensuels et avec l'appui du Centre Départemental de Loire-Atlantique de Météo-France, une analyse météorologique des journées pluvieuses considérées a pu être effectuée au niveau de notre zone d'étude. Cette analyse, associée à la visualisation des images radar, a permis de séparer ces 13 journées en 24 périodes pluvieuses. Ces périodes sont temporellement continues et sont homogènes visuellement en terme de type de pluie. Elles ont une durée comprise entre 35 minutes et 3 heures.

Dans un second temps, les 24 périodes pluvieuses ont été réparties en quatre groupes. Le Groupe 1 rassemble neuf périodes pluvieuses d'intensité faible pouvant être associées à un secteur chaud. Le Groupe 2 regroupe sept périodes d'averse associées à des ciels de traine. Les quatre périodes du Groupe 3 sont des systèmes orageux peu organisés et les quatre périodes du Groupe 4 sont des systèmes orageux organisés en bandes pluvieuses.

La représentativité des données radar a été appréciée de deux façons différentes. Tout d'abord, Météo France calcule en temps réel un facteur de calibrage en comparant les données radar aux données de pluviomètres situés entre 0 et $100 \mathrm{~km} \mathrm{du}$ radar [3]. Ce facteur de calibrage (i.e. le rapport moyen entre les données pluviométriques et les données radar), moyenné de façon journalière, varie pour les journées considérées entre 0.73 et 1.01 avec une valeur de 1.35 pour une des journées. Si les données journalières sont du même ordre de grandeur, des variations significatives peuvent être notées au pas de temps horaire. Un test supplémentaire a été effectué en comparant les données radar à celles de quatre pluviomètres au pas de temps horaires. Ces quatre pluviomètres sont localisés entre 8 et $15 \mathrm{~km}$ du radar et sont gérés par notre groupe de recherche. Les rapports moyennés journalièrement sont cohérents avec les valeurs des rapports précédents tout en présentant une gamme de variation plus élevée, entre 0.56 et 1.06 , avec une valeur de 1.66 pour une des journées. Ces rapports présentent également une importante variabilité temporelle ainsi qu'une variabilité spatiale au pas de temps horaire, malgré la proximité des pluviomètres (entre 1 et $10 \mathrm{~km}$ ). Dans ces conditions, nous avons préféré conserver les images radar inchangées afin de valider la classification effectuée. De plus, l'analyse structurale a été faite en adoptant une approche climatologique, c'est-àdire en travaillant sur les variations relatives de la pluie ( $c f$. Partie III.) ceci présentant l'avantage de s'affranchir du biais de la mesure radar.

\section{II.2.2. Validation de la séparation des journées en périodes pluvieuses et en groupes}

Deux analyses différentes ont été effectuées afin de vérifier la pertinence de la séparation des journées en périodes pluvieuses et du regroupement de ces périodes en quatre groupes.

Une Analyse en Composantes Principales normée (ACP) [6] a tout d'abord été réalisée sur les images radar de l'ensemble des périodes pluvieuses (soit un total de 405 images radar). Pour chacune des images radar, quatre variables ont été calculées : le pourcentage de zéros et, sur les valeurs de pluie strictement positives, la moyenne, l'écart-type et la médiane. Seuls les deux premières composantes principales (axes) ont été retenues du fait qu'elles représentent à elles seules plus de $94 \%$ de la variance totale.

La Figure 2a) représente la projection des quatre variables sur les deux premiers axes. La moyenne et l'écart-type sont fortement négativement corrélés avec l'axe 1 (corrélation respective de 0.98 et 0.94 ). La médiane est positivement corrélée avec l'axe 2 (corrélation de 0.67 ) et négativement corrélée avec l'axe 1 (corrélation de 0.68 ). Le pourcentage de zéro est quant à lui corrélé négativement avec les deux axes (corrélation de 0.52 avec l'axe 1 et de 0.81 avec l'axe 2).

La Figure 2b) illustre les résultats de l'ACP. On peut tout d'abord observer que les périodes pluvieuses choisies sont bien constituées d'images radar de fortes ressemblances. Par 
ailleurs, les images radar du Groupe 1 sont situées au niveau des coordonnées positives de l'axe 1 : ces images sont ainsi caractérisées par une moyenne et une écart-type plus faibles que ceux des autres groupes. En comparaison avec les autres groupes, les images du Groupe 2 ont, une moyenne et un écart-type élevés associés à un fort pourcentage de zéros. Globalement, les images des Groupes 3 et 4 ont une médiane supérieure associée à une moyenne et à un écart-type élevés. Cependant leur séparation en deux groupes distincts n'apparait pas justifiable d'après l'ACP considérée.

Une seconde analyse, basée sur un descripteur autre que le pourcentage de zéros, la moyenne, l'écart-type ou la médiane, s'est donc avérée nécessaire. Dans ce but, la distance minimale séparant chaque pixel d'intensité strictement positive d'un pixel d'intensité nulle a été calculée pour chaque image radar appartenant aux Groupes 3 et 4 . De cette manière, les périodes pluvieuses du Groupe 3 se différencient nettement de celles du Groupe 4. En effet, les amas pluvieux du Groupe 3 apparaissent peu organisés, avec des intensités maximales localisées aussi bien au bord qu'au centre des amas. Au contraire, les amas pluvieux du Groupe 4 sont davantage organisés avec des intensités maximales majoritairement localisées dans la partie centrale des amas.

Ainsi, les deux études précédentes ont montré l'homogénéité des périodes pluvieuses sélectionnées. Par ailleurs, elles ont confirmé que les quatre groupes formés différaient les uns des autres et que chacun d'eux était constitué de périodes pluvieuses présentant les mêmes caractéristiques. La prochaine partie traite de l'analyse de la variabilité des champs pluvieux par une approche géostatistique.

\section{ANALYSE STRUCTURALE}

\section{III.1. Présentation}

La géostatistique a d'abord été utilisée dans le domaine minier [7] mais ses champs d'application se sont rapidement étendus à d'autres domaines tel celui des sciences de l'eau [8]. Plusieurs études ont montré que les outils géostatistiques s'avéraient efficaces pour caractériser la variabilité des précipitations [9]. Les travaux concernant ce sujet ont été réalisés essentiellement à partir de réseaux de pluviomètres. Les données radar commencent à être utilisées pour la caracté- risation de la structure des précipitations en contexte urbain [4] ou de crues « éclair».

Le variogramme, $\gamma$, permet de caractériser la structure spatiale et temporelle de la fonction aléatoire $Z$ dont le champ pluvieux est une réalisation. Il s'exprime de la façon suivante :

$$
\gamma(d)=\frac{1}{2} E\left[(Z(x)-Z(x+d))^{2}\right],
$$

où $E$ représente l'espérance mathématique et $d$ le décalage spatial ou temporel. Le variogramme apporte des informations sur la continuité et sur la cofluctuation des données en fonction de leur éloignement.

Une période pluvieuse peut être associée à un contexte de multi-réalisations, chaque image radar est alors considérée comme une réalisation. Lorsque le phénomène étudié possède une variance définie, le variogramme atteint un palier à une portée donnée et, dans ce cas-là, le palier est égal à la variance. On peut alors normaliser le variogramme, par la variance respective de chaque réalisation, puis le moyenner sur l'ensemble des réalisations. On obtient ainsi un variogramme dit «climatologique» ([4]; [10]), caractérisé par un palier égal à 1 , mais en perdant l'information quant à la variance. Cependant, ce variogramme fournit une indication quant à la structure représentative d'un ensemble de champs en donnant un poids équivalent à chacun d'entre eux. Il permet également de s'affranchir du biais de mesure des données radar. La portée, quant à elle, correspond à la distance de décorrélation, c'est-à-dire à la distance à partir de laquelle deux points de mesure ont un comportement statistiquement indépendant. Finalement, si le variogramme ne présente pas de discontinuité à l'origine (également appelé «effet pépite »), ceci montre que l'échelle utilisée est suffisamment fine pour décrire la variabilité du phénomène étudié.

Dans ce travail nous avons analysé le variogramme de la Pluie Non Nulle (PNN) afin d'étudier la variabilité au sein même des amas pluvieux. Dans le calcul de ce variogramme, seuls les points de mesure ayant une intensité de pluie strictement supérieure à zéro ont été pris en compte.

Les variogrammes climatologiques spatiaux multidirectionnels et temporels, ont été étudiés via l'analyse de leur forme et de leur portée. Concernant le variogramme temporel, deux approches ont été considérées : l'approche eulé-
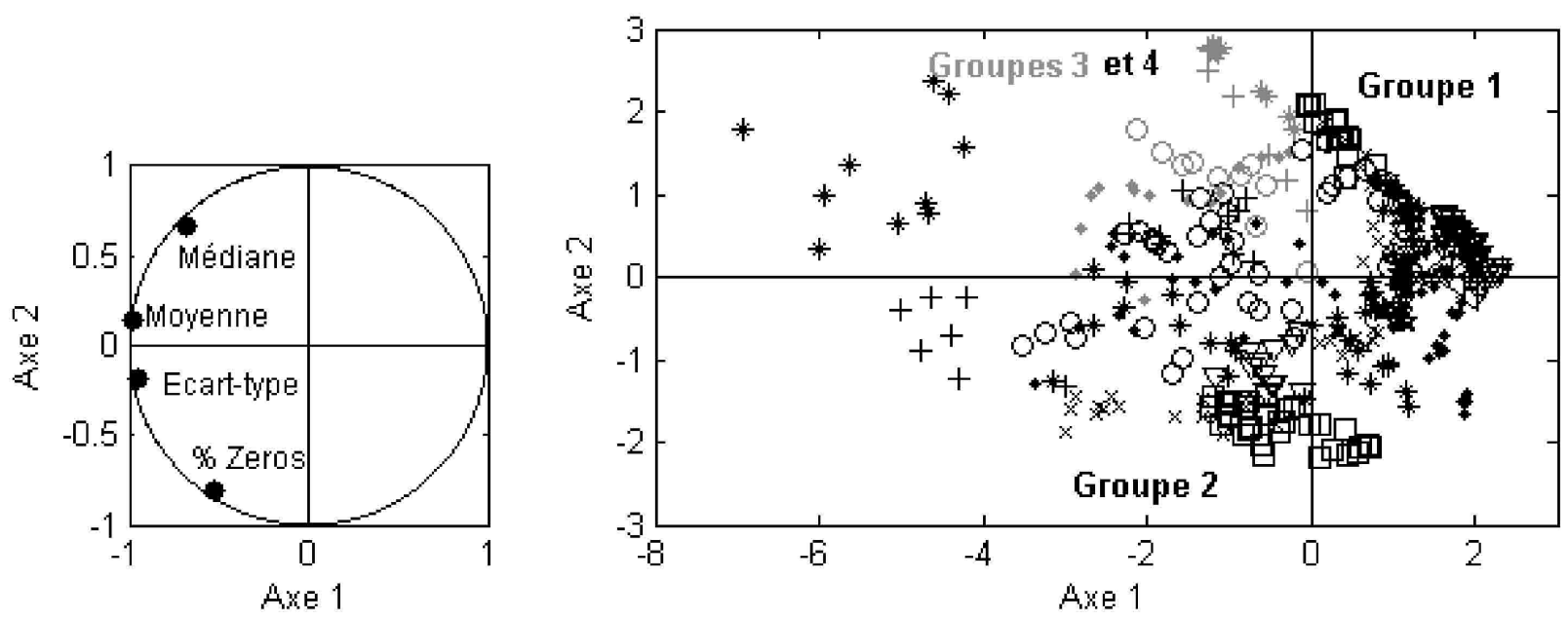

Figure 2 : a) projection sur les deux premiers axes des 4 variables considérées (à gauche). Le cercle de corrélation est représenté. b) projection des individus (images radar) des 4 groupes sur les deux premiers axes (à droite). A l'intérieur de chaque groupe, les individus d'une même période pluvieuse sont représentés avec un sigle identique. 
rienne et l'approche lagrangienne. L'approche eulérienne est effectuée à une position fixe et regarde défiler le champ pluvieux. Au contraire, l'approche lagrangienne suit le déplacement du champ pluvieux. Le variogramme eulérien fournit donc une information combinée sur la structure spatio-temporelle du champ pluvieux tandis que le variogramme lagrangien permet d'étudier uniquement la composante temporelle de la variabilité du champ de pluie.

Les variogrammes spatiaux multidirectionnels et temporels de la PNN ont été calculés pour les quatre groupes et ont été étudiés à travers leur forme et leur portée.

\section{III.2. Structure spatiale et temporelle de la Pluie Non Nulle}

\section{III.2.1. Structure spatiale}

Les variogrammes climatologiques instantanés spatiaux multidirectionnels de la PNN de chacun des quatre groupes sont rassemblés Figure 3.
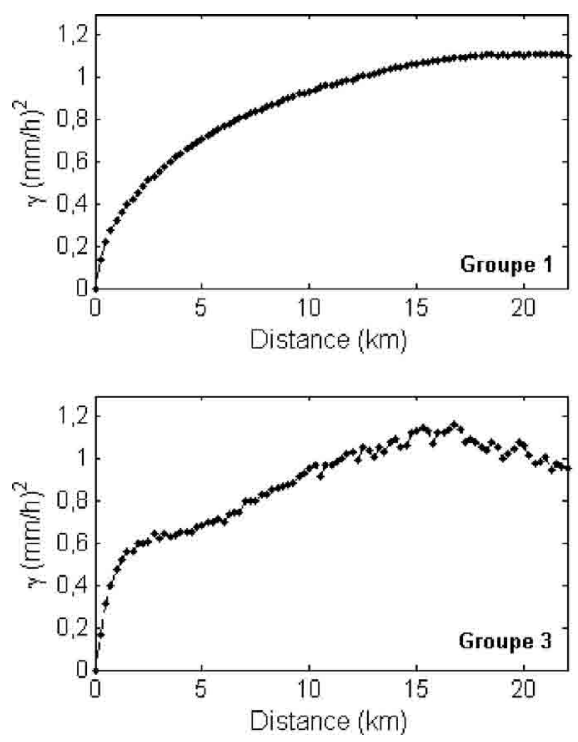

Les variogrammes des Groupes 1 et 2 présentent une structure unique de portée respective de 17 et $5 \mathrm{~km}$. Cela signifie, par exemple, que pour les périodes pluvieuses du Groupe 2, deux points distants de $5 \mathrm{~km}$ ont un comportement indépendant. Le variogramme du Groupe 3 est particulier puisqu'il possède deux structures et décroît une fois atteint le second palier. La première structure correspond à une distance de décorrélation d'environ $2.5 \mathrm{~km}$ tandis que la seconde correspond à une portée de $15 \mathrm{~km}$. De même, le variogramme du Groupe 4 est atypique : il atteint une valeur maximale à $14 \mathrm{~km}$ puis la variabilité décroît. Aucune explication n'a pu être trouvée permettant d'interpréter la décroissance des variogrammes des Groupes 3 et 4 lors de décalages élevés. Une décomposition des images radar en deux sous-processus nous a cependant permis de retrouver des formes standards de variogrammes ( $c f$. Partie III.2.3).

On peut noter que ces variogrammes ne présentent pas d'effet de pépite et qu'ils sont caractérisés, même les variogrammes de portée élevée (Groupes 1 et 4) par une mon-
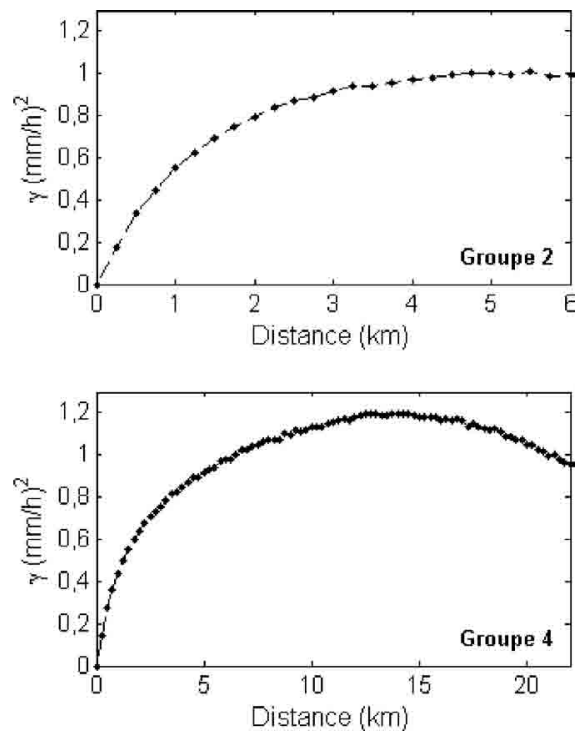

Figure 3 : Variogrammes climatologiques instantanés spatiaux multidirectionnels de la PNN des quatre groupes, zoomés afin de mieux visualiser la montée et la forme de chaque variogramme.
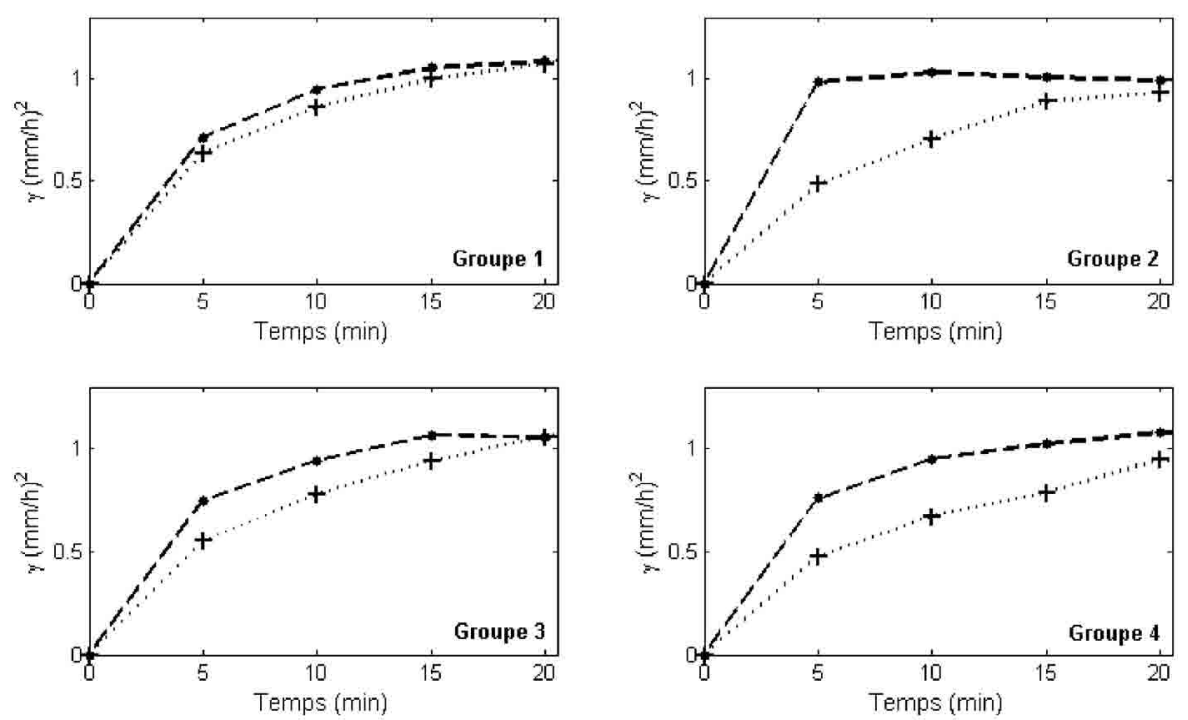

Figure 4 : Variogrammes climatologiques instantanés temporels de la PNN des quatre groupes : eulériens (points) et lagrangiens (plus). 
tée très rapide. Chacun des variogrammes atteint $50 \%$ de leur palier pour une distance inférieure à $5 \mathrm{~km}$. Ce résultat semble intéressant car il témoigne de la forte variabilité spatiale des champs de pluie instantanés à courte distance et ceci pour tous les types de pluie.

\section{III.2.2. Structure temporelle}

Les variogrammes climatologiques instantanés temporels eulériens et lagrangiens de la PNN des quatre groupes sont présentés en Figure 4.

Les variogrammes eulériens des Groupes 1 et 2 possèdent une portée unique de respectivement 15 et $5 \mathrm{~min}$. Même si les variogrammes spatiaux des Groupes 3 et 4 sont particuliers, les variogrammes temporels présentent une portée unique de l'ordre de 15 et $20 \mathrm{~min}$.

Les variogrammes lagrangiens sont caractérisés par des variabilités inférieures à celles des variogrammes eulériens et par des temps de décorrélation d'au moins $20 \mathrm{~min}$. Ceci peut s'expliquer par le fait que les variogrammes lagrangiens ne prennent en compte que la seule dimension temporelle alors que les variogrammes eulériens incluent les dimensions spatio-temporelles. Les variogrammes lagrangiens ne permettant pas de distinguer les différents groupes, seuls les variogrammes eulériens ont été utilisés par la suite.

Cette analyse variographique apparaît donc cohérente avec les observations météorologiques effectuées ( $c f$. Partie II.2.1). Les périodes pluvieuses du Groupe 1 sont relativement peu variables sur le domaine étudié (distance de décorrélation de la PNN de $17 \mathrm{~km}$ ). Le Groupe 2 est constitué de périodes pluvieuses composées de petits amas variables (distance de décorrélation de la PNN de $5 \mathrm{~km}$ ). Enfin, les structures particulières des variogrammes spatiaux des Groupes 3 et 4 pourraient s'expliquer par la présence de petits amas pluvieux, plus variables, eux-mêmes situés au sein d'amas moins variables d'extension spatiale plus importante.

\section{III.2.3. Cas particulier des doubles structures}

Nous avons tenté de séparer, pour chacun des Groupes 3 et 4 , les pixels de chaque image radar en deux classes, la première classe correspondant aux petites structures variables et la seconde aux amas moins variables. Dans ce but, nous avons utilisé l'algorithme de classification de Kohonen [11] permettant le regroupement des pixels en différentes classes à la fois les plus homogènes et les plus distinctes possibles. Cet algorithme a pour caractéristique d'organiser les différentes classes (qu'il construit) dans un espace de représentation prédéfini (une sphère dans notre cas), dans lequel la priorité est donnée à la ressemblance entre classes voisines [12]. La visualisation des images radar laisse penser que les petits amas variables sont caractérisés par des intensités supérieures à celles des amas moins variables. Ainsi, chaque pixel radar est décrit par deux variables : d'une part, l'intensité moyenne de ses huit voisins et, d'autre part, l'écart-type associé (indicatif de la variabilité). Une première classification des pixels radar en 25 classes a ainsi pu être effectuée suivant ces deux variables. Dans un second temps, ces classes ont été regroupées en 2 "superclasses » [12] : la première réunit les pixels les plus intenses et variables (classe 1) et, la seconde, les pixels les moins intenses et les moins variables (classe 2). Il est intéressant de noter que les deux variables utilisées (intensité moyenne et écart-type

Cette classification a été effectuée pour chaque période pluvieuse des Groupes 3 et 4 et tous les pixels radar d'intensité strictement positive ont pu être rattachés à l'une des deux classes. Les variogrammes ont ensuite été calculés en suivant la même approche que précédemment, en ne considérant d'abord que les pixels appartenant à la première classe puis, ensuite, en ne considérant que les pixels de la seconde classe (Figure 5).

L'analyse variographique des pixels des classes 1 et 2 montre ainsi que les périodes pluvieuses du Groupe 3 sont constituées d'amas, de portée spatiale d'environ $1.5 \mathrm{~km}$ et de 5 min de temps de décorrélation (classe 1), associés à des amas moins variables caractérisés par des portées de $12 \mathrm{~km}$ et des temps de décorrélation de 15 min (classe 2). De même, deux structures ont pu être identifiées au cours des périodes pluvieuses du Groupe 4. La première se caractérise par des amas de portées d'environ $5 \mathrm{~km}$ et 5 min (classe 1) et, la seconde, par des amas de portées d'environ $15 \mathrm{~km}$ et $15 \mathrm{~min}$ (classe 2). On rappelle que la classe 1 regroupe les pixels intenses, les pixels les moins intenses constituant la classe 2. Il est intéressant de noter que là encore les variogrammes présentent une forte variabilité spatiale à courte distance. Les variogrammes spatiaux de la classe 1 atteignent notamment $50 \%$ de leur palier à une distance inférieure à $1 \mathrm{~km}$. Par
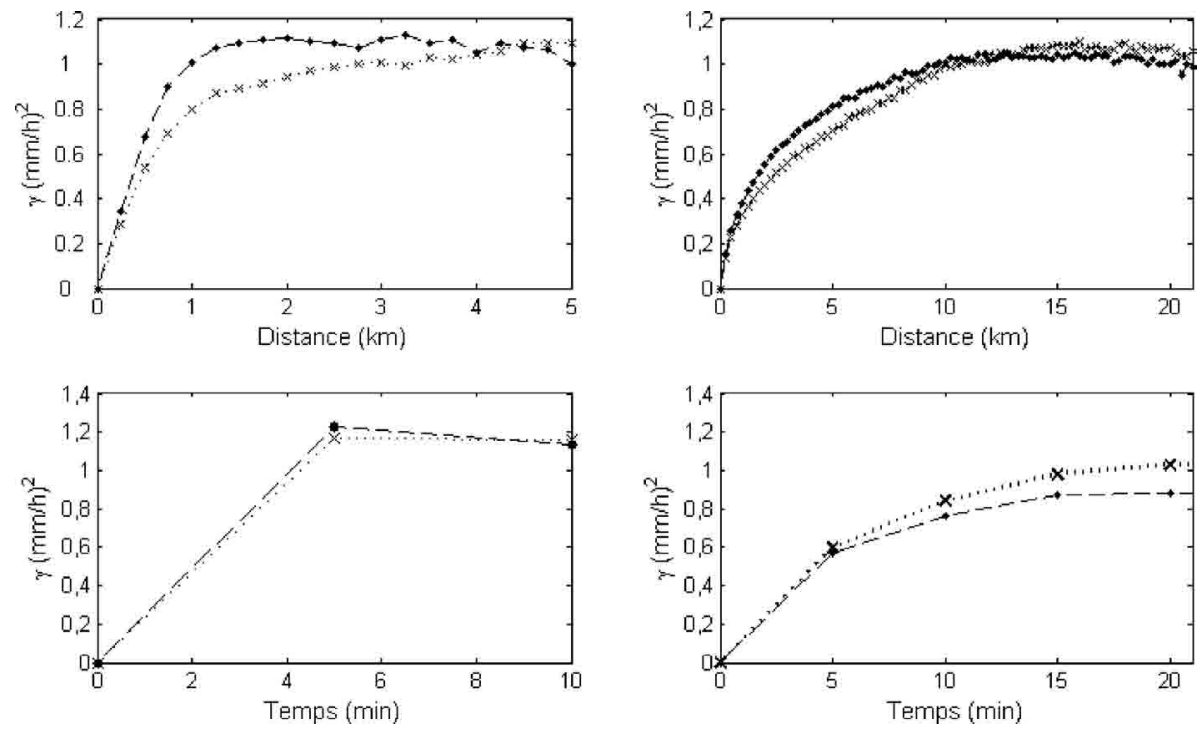

Figure 5 : Variogrammes climatologiques instantanés spatiaux multidirectionnels (en haut) et temporels eulériens (en bas) de la PNN de la classe 1 (à gauche) et de la classe 2 (à droite) pour le Groupe 3 (points) et le Groupe 4 (croix). 
ailleurs, on peut observer que variogrammes des classes 1 et 2 sont proches entre les deux groupes.

\section{CONCLUSIONS}

L'objectif de ce travail était d'analyser la variabilité spatiale et temporelle de la Pluie Non Nulle de champs pluvieux à des échelles spatio-temporelles cohérentes avec les applications hydrologiques urbaines. Les données utilisées étaient des images radar de résolution temporelle instantanée et de résolution spatiale de $250 \times 250 \mathrm{~m}^{2}$. Ces images radar ont été enregistrées, entre mai et novembre 2009, au cours de 13 journées pluvieuses par le radar météorologique de Treillières (France). Une analyse météorologique de ces 13 journées a permis d'en extraire 24 périodes pluvieuses qui ont été regroupées en quatre groupes. Chaque groupe est homogène en termes de type de pluie. Deux analyses ont confirmé l'homogénéité de chaque groupe.

L'étude des variogrammes de Pluie Non Nulle a permis de mettre en évidence quatre structures pluvieuses présentant des échelles de variabilité très différentes. La première structure est caractérisée par une distance de décorrélation de l'ordre de $17 \mathrm{~km}$ et un temps de décorrélation de $15 \mathrm{~min}$ et correspond à des zones pluvieuses peu variables. La deuxième se distingue par une distance de décorrélation de $5 \mathrm{~km}$ et un temps de décorrélation de 5 min et correspond à des amas pluvieux intenses et fortement variables (averses). Les troisième et quatrième structures sont des doubles structures (orages). Elles sont constituées par l'association de petits amas intenses (portées de 5 min et inférieures à $5 \mathrm{~km}$ ) localisées au sein d'amas moins variables (portées de 12-15 km et $15 \mathrm{~min}$ ). Ces structures diffèrent principalement par leur niveau d'organisation; la troisième structure apparait peu organisée alors que la quatrième a été identifiée comme des bandes pluvieuses d'intensités maximales localisées en leur centre. Cette étude a également montré que les variogrammes spatiaux de l'ensemble des groupes présentaient une montée très rapide et atteignaient $50 \%$ de leur palier à des distances inférieures à $5 \mathrm{~km}$. Ainsi, cette étude montre l'intérêt d'utiliser des images radar de haute résolution capable de détecter ces fortes variabilités à courtes distances.

Ce travail a étudié un nombre restreint de périodes pluvieuses disponibles au début de cette étude. Cependant la méthodologie utilisée et présentée dans cet article pourrait être appliquée à un nombre plus important de périodes pluvieuses afin d'obtenir une description détaillée de la structure de champs pluvieux en fonction de leur typologie météorologique. Il serait également intéressant d'analyser comment chaque type de pluie affecte la modélisation hydrologique pluie-débit sur de petits bassins versants urbains réagissant en quelques dizaines de minutes au plus. Finalement, ces résultats associés à l'analyse des variogrammes d'indicateur pourraient être utilisés pour simuler des champs pluvieux de variabilité donnée [13].

\section{REMERCIEMENTS}

Les auteurs souhaitent vivement remercier Météo France, pour la mise à disposition des images radar, et particulièrement M. Abdel-Amin Boumahmoud. Les auteurs remercient également M. Christophe Pin, du Centre Départemental de Loire-Atlantique de Météo France, pour son aide quant à l'analyse météorologique des journées étudiées.

\section{REFERENCES ET CITATIONS}

[1] Segond M.-L., Wheater H.S., \& Onof C. (2007) - The significance of spatial rainfall representation for flood runoff estimation: A numerical evaluation based on the Lee catchment, UK. Journal of Hydrology. 347 116-131

[2] Krajewski W.F., Villarini G., \& SMith J.A. (2010) — RadarRainfall uncertainties - Where are we after thirty years of efforts ?. B. Am. Meteorol. Soc. 91 87-94

[3] Emmanuel I., Andrieu H., \& Tabary P. (2011) - Evaluation of the new French operational radar product for the field of urban hydrology. Atmospheric Research, en révision

[4] Berne A., Delrieu G., Creutin J.-D., \& Obled C. (2004) Temporal and spatial resolution of rainfall measurements required for urban hydrology. Journal of Hydrology. 299 166-179

[5] TABARY P. (2007) - The new French operational radar rainfall product. Part 1: methodology. Weather and Forecasting. 22 393-408

[6] Pearson K. (1901) - On Lines and Planes of Closest Fit to Systems of Points in Space. Philosophical Magazine. 6 (2) 559-572

[7] Matheron G. (1965) - Les Variables Régionalisées et leur estimation. Masson et Cie, Paris.

[8] Delhomme J. (1978) - Kriging in the hydrosciences. Advances in Water Ressources. 1 (5) 251-266

[9] Barancourt C., Creutin J.D., \& Rivoirard J. (1992) - A Method for Delineating and Estimating Rainfall Fields. Water Resources Research. 28 1133-1144

[10] Borga M., \& Vizzaccaro A. (1997) - On the interpolation of hydrologic variables : formal equivalence of multiquadratic surface fitting and kriging. Journal of Hydrology. 195 160-171

[11] KoHonen T. (2001) - Self-Organizing Maps. Springer, New York.

[12] Bennani Y. (2006) - Apprentissage connexionniste. Lavoisier, Paris.

[13] Leblois E. (2011) - Technical presentation of the Turning Band Method precipitation simulation system. Communication personnelle. 